\title{
Estimating Temperature Fluctuations in the Early Universe
}

\author{
Debashis Gangopadhyay * \\ S. N. Bose National Centre for Basic Sciences, JD-Block, Sector-III, Salt Lake, Kolkata 700098, India \\ and Centre for Astroparticle Physics and Space Science, \\ Bose Institute (Salt Lake Campus), EN-Block, Salt Lake, Kolkata-700091, India
}

\begin{abstract}
A lagrangian for the $k$ - essence field is constructed for a constant scalar potential and its form determined when the scale factor was very small compared to the present epoch but very large compared to the inflationary epoch. This means that one is already in an expanding and flat universe. The form is similar to that of an oscillator with time-dependent frequency. Expansion is naturally built into the theory with the existence of growing classical solutions of the scale factor. The formalism allows one to estimate fluctuations of the temperature of the background radiation in these early stages (compared to the present epoch) of the universe. If the temperature at time $t_{a}$ is $T_{a}$ and at time $t_{b}$ the temperature is $T_{b}\left(t_{b}>t_{a}\right)$, then for small times, the probability for the logarithm of inverse temperature evolution can be estimated to be given by
\end{abstract}

$$
\begin{gathered}
P(b, a)=\left|\left\langle\ln \left(\frac{1}{T_{b}}\right), t_{b} \mid \ln \left(\frac{1}{T_{a}}\right), t_{a}\right\rangle\right|^{2} \\
\approx\left(\frac{3 m_{\mathrm{P} l}^{2}}{\pi^{2}\left(t_{b}-t_{a}\right)^{3}}\right)\left(\ln T_{a}\right)^{2}\left(\ln T_{b}\right)^{2}\left(1-3 \gamma\left(t_{a}+t_{b}\right)\right)
\end{gathered}
$$

where $0<\gamma<1, m_{\mathrm{P} l}$ is the Planck mass and Planck's constant and the speed of light has been put equal to unity. There is the further possibility that a single scalar field may suffice for an inflationary scenario as well as the dark matter and dark energy realms.

Keywords: physics of the early universe,inflation, dark matter theory, dark energy theory.

PACS numbers: 95.35.+d ; 95.36.+x ; 98.80.Cq ; 98.80.-k

\section{Introduction}

An approach to understand the origins of dark matter and dark energy involve setting up lagrangians for what are known as $k$-essence fields. Usually one takes the Friedman-Robertson-Walker metric with zero curvature constant so that the universe is flat. Recently a lagrangian for the $k$ - essence field has been set up [1] where there are two generalised coordinates $q(t)=\ln a(t)(a(t)$ is the scale factor) and a scalar field $\phi(t)$ with a complicated polynomial interaction between them. In the lagrangian, $q$ has a standard kinetic term while $\phi$ does not have a kinetic part and occurs purely through the interaction term. Classical solutions of this lagrangian give very good results for the cosmological parameters [1]. It had been shown previously [2] that it is possible to unify the dark matter and dark energy components into a single scalar field model with the scalar field $\phi$ having a non-canonical kinetic term. These scalar fields are the $k$-essence fields which first appeared in models of inflation [3] and subsequently led to models of dark energy also [4]. The general form of the lagrangian for these $k$-essence models is assumed to be a function $F(X)$ with $X=\nabla_{\mu} \phi \nabla^{\mu} \phi$, and do not depend explicitly on $\phi$ to start with. In [2], $X$ was shown to satisfy a general scaling relation, viz. $X\left(\frac{d F}{d X}\right)^{2}=C a(t)^{-6}$ with $C$ a constant (similar expression was also derived in 5]). Using this, in 2] specific forms (motivated from string theory [6], 3], 12]) were assumed for the lagrangian and $F(X)$ to show that self-consistent models can be built which account for both the dark matter and dark energy components. 1] incorporates the scaling relation of [2]. Literature on dark matter, dark energy and $k$ - essence can be found in [7], [8], [9], 10], [11].

The motivation for this work comes from the fact that as classical solutions of the lagrangian in [1] give realistic results, addressing questions relating to (quantum) fluctuations becomes meaningful. In fact, questions regarding the amplitude of a scale factor at some epoch evolving to a different value at a later epoch can surely be addressed for a lagrangian with canonical kinetic terms. These effects are relevant at times close to the big bang (very small scale factor). However, then the curvature constant is not zero. To get past this difficulty, in this work, an epoch is chosen when the scale factor was small compared to the present epoch but very large compared to the big bang and inflationary epoch. This means that one is already in an expanding and flat universe- however,the influence of inflation is still around.

In this work, the scalar potential $V(\phi)=V$ is a (positive) constant and all the time variables $t \equiv t / t_{0}$, where $t_{0}$ is the present epoch and we are interested only in $t<1$ scenarios. Also $a\left(t_{1}\right)<a\left(t_{2}\right)$ for $t_{1}<t_{2}$ etc. So one has to find how the lagrangian looks at smaller values of the scale factor,find possible classical solutions and then find relevant fluctuations. As the scale factor is inversely proportional to the temperature at a particular epoch, these amplitudes also provide an estimate of temperature fluctuations. In this work, I discuss a plausible 
formalism for this and show that the classical solutions in this domain are fully consistent with the inflationary scenario and a radiation dominated universe.Moreover, a single scalar field may suffice for an inflationary scenario as well as the dark matter and dark energy realms and a measure of temperature fluctuations may be estimated using standard prescriptions.

2. Lagrangian in the early universe

The lagrangian $L$ (or the pressure $p$ ) is taken as

$$
L=-V(\phi) F(X)
$$

The energy density is

$$
\rho=V(\phi)\left[F(X)-2 X F_{X}\right]
$$

with $F_{X} \equiv \frac{d F}{d X}$ and in the present work $V(\phi)=V$ is a constant $(>0)$. Before proceeding further, a digression on the origin of the equation (1) is in order. The present view is that dark energy can account for the observed acceleration of the galaxies. One can show that dark energy with sufficiently negative pressure will accelerate the expansion of the universe once it starts dominating over matter. A simple fluid model with negative presure is the cosmological constant with equation of state parameter $w=-1$, and energy density $\rho=-p$, the pressure. If one identifies the dark energy with a cosmological constant then a fundamental length is introduced into the theory [7] $L_{\Lambda} \equiv H_{\Lambda}^{-1}$ related to the dark energy density $\rho_{D E}$ by $H_{\Lambda}^{2} \equiv \frac{8 \pi G \rho_{D E}}{3}$. Observations then require that $\left(L_{\mathrm{P} l}^{2} / L_{\Lambda}^{2}\right) \leq 10^{-123}$, where $L_{\mathrm{Pl}}$ is the Planck length. So there is an enormous fine tuning problem. There are also other problems. Alternative possibilities entail use of scalar fields $\phi$ with suitably chosen potential $V(\phi)$ so as to have variable (with time) vacuum energy. The hope is then that fine tunings can be avoided with suitably chosen potentials. Two such models with variable $w$ are $L_{\text {quintessence }}=\frac{1}{2} \partial_{\mu} \phi \partial^{\mu} \phi-V(\phi)$ and $L_{\text {tachyon }}=$ $-V(\phi)\left[1-\partial_{\mu} \phi \partial^{\mu} \phi\right]^{\frac{1}{2}}$. The quintessence model can be shown to be a natural generalisation of the lagrangian for a non-relativistic particle [7]. In a cosmological context, the quintessence (scalar field) models leads to potential energy driven acceleration $\left(V(\phi)>\partial^{\mu} \phi \partial_{\mu} \phi\right)$. The tachyon lagrangian originated from string theory [12] and can be shown to be a field theoretic analogue of the lagrangian of a relativistic particle [7]. The model allows solutions where $V \rightarrow 0, \partial_{i} \phi \partial^{i} \phi \rightarrow 1$ simultaneously, keeping the energy density finite. So here the kinetic energy dominates and in the cosmological context can give rise to acceleration. These are the $k$-essence models for dark energy. In the cosmological scenario, the $\left[1-\partial_{\mu} \phi \partial^{\mu} \phi\right]^{\frac{1}{2}}$ factor is replaced by a more general function $F(X)$ with $X=\partial^{\mu} \phi \partial_{\mu} \phi$. This is the genesis of equation (1).

For a flat Robertson Walker metric the equation for the $k$-essence field is

$$
\left(F_{X}+2 X F_{X X}\right) \ddot{\phi}+3 H F_{X} \dot{\phi}+\left(2 X F_{X}-F\right) \frac{V_{\phi}}{V}=0
$$

$H=\frac{\dot{a}(t)}{a(t)}$ is the Hubble parameter. For a homogeneous and isotropic universe $\phi(x, t) \equiv \phi(t)$, and so $X=\frac{1}{2} \dot{\phi}^{2}$. In [2], $V(\phi)=$ const. so $V_{\phi} \equiv \frac{d V}{d \phi}=0$ and (3a) becomes

$$
\left(F_{X}+2 X F_{X X}\right) \dot{X}+6 H F_{X} X=0
$$

Changing the independent variable from the time $t$ to the scale factor $a$ i.e. $\frac{d X}{d t}=a H \frac{d X}{d a}$ leads to [2]

$$
\left(F_{X}+2 X F_{X X}\right) a \frac{d X}{d a}+6 F_{X} X=0
$$

whose solution is the scaling law [2] :

$$
X F_{X}^{2}=C a^{-6}
$$

In this work also we take $V(\phi)=V=$ constant so that the above scaling law holds.

Using (4) and the zero-zero component of Einstein's field equations an expression for the lagrangian is obtained as follows.Take the Robertson-Walker metric :

$$
d s^{2}=c^{2} d t^{2}-a^{2}(t)\left[\frac{d r^{2}}{\left(1-k r^{2}\right)}+r^{2}\left(d \theta^{2}+\sin ^{2} \theta d \phi^{2}\right)\right]
$$

where $k=0,1$ or -1 is the curvature constant. The zero-zero component of Einstein's equation reads:

$$
R_{00}-\frac{1}{2} g_{00} R=-\kappa T_{00}
$$

This gives with the metric (5)

$$
\frac{k}{a^{2}}+H^{2}=\frac{8 \pi G}{3} \rho
$$

Using (1), (3), (6) and (7), and taking $k=0$, we arrive at

$$
X F_{X}=\frac{1}{2 X}\left[F-\left(\frac{3}{8 \pi G V)}\right) H^{2}\right]
$$

Using (4) to eliminate $F_{X}$ gives

$$
F(X)=2 \sqrt{C} \sqrt{X} a^{-3}+3 \frac{H^{2}}{8 \pi G V}
$$

So the expression for the lagrangian is obtained as

$$
\begin{gathered}
L=-2 \sqrt{C} \sqrt{X} a^{-3} V-\left(\frac{3}{8 \pi G}\right) H^{2} \\
=-2 \sqrt{C} \sqrt{\dot{\phi}^{2}-(\nabla \phi)^{2}} a^{-3} V-3 \frac{H^{2}}{8 \pi G}
\end{gathered}
$$

Homogeneity and isotropy of spacetime imply $\phi(t, \mathbf{x})=$ $\phi(\mathbf{t})$. Then (10) becomes

$$
L=-c_{1} \dot{q}^{2}-c_{2} V \dot{\phi} e^{-3 q}
$$

where $a(t)=e^{q(t)}, c_{1}=3(8 \pi G)^{-1}, c_{2}=2 \sqrt{C}$, (we shall always take the positive square root of $C$ ) and the scalar potential $V$ is a constant. 
The lagrangian (11) has a kinetic term for $q$. The other term is an interaction term and there is no kinetic term for $\phi$. Note that $a=e^{q}$. It is readily seen from the graph of the exponential function (in figure $1 x \equiv q$ ) that in the region $-1<q<0$ one has , $a=e^{q}<1$. Hence in this region $q$ small (i.e. $|q|<1$ ) means $a$ is also small (i.e $a<1$ ). Moreover, in this region $a$ grows from $e^{-1}=0.367879$ to $e^{0}=1$. So within this region $a$ grows as $q$ grows. So smaller values of $q$ mean that we are going back to smaller values of $a$ i.e. to earlier epochs. In this work we will restrict ourselves to this domain. In this domain expand the exponential in (11) :

$$
L=-c_{1} \dot{q}^{2}-[2 \sqrt{C} V \dot{\phi}]\left[1-3 q+\frac{9}{2} q^{2}+\ldots\right]
$$

Keeping terms upto $O\left(q^{2}\right)$ and replacing $q$ by $q+\frac{1}{3}$ one has

$$
L=-\frac{M}{2}\left[\dot{q}^{2}+12 \pi G g(t) q^{2}\right]-\left(\frac{1}{2}\right) g(t)
$$

where $M=\frac{3}{4 \pi G}=\frac{3 m_{\mathrm{P} l}^{2}}{4 \pi}, g(t)=2 \sqrt{C} V \dot{\phi}, m_{\mathrm{P} l}$ is the Planck energy and we use $\hbar=c=1$ ( $c$ is speed of light, $\hbar$ is Planck's constant).

Here two clarifications must be made. Firstly, $q=\ln a$ means $\dot{q}=\frac{\dot{a}}{a}=H(t)$. In the present work the Hubble parameter $H(t)$ is not a constant but a function of time as it usually is.

Secondly,although in (12) the lagrangian is an explicit function of $a, \dot{a}$, it would be incorrect to assume that one should have this imposed right from equation (1) itself. This is because the dependence on $a, \dot{a}$ follows only after the following operations have been carried out , viz.,

(a) the Robertson-Walker metric is chosen and the equation for the $k$ - essence field is set up for a constant $V(\phi)$;

(b)a change of the independent variable is made from the time $t$ to the scale factor $a$;

(c) the scaling law (4) obtained;

(d) the zero-zero component of Einstein's equation incorporated to relate the energy density to the curvature constant $k$ (subsequently we take $k=0$ ) and the Hubble parameter; and

(e) the scaling law (4) used to eliminate the derivative $F_{X}$.

Therefore, one must start with an independent potential in the lagrangian and let the relevant equations dictate (a posteriori) the final form for the lagrangian. In this method there is no possibility of non-Einsteinian gravity equations emerging. It is shown below (equations (21),(22)) that the the classical solution for the scalar field is linear in the time $t$ so that in $L_{\mathrm{cl}}$ the term proportional to $\dot{\phi}_{\mathrm{cl}}$ is effectively a constant without any dynamical consequences. In fact, all total derivatives will be ignored (see below).

\section{Chaotic Inflation}

First consider a possible classical solution in this model. Put $12 \pi G g(t)=-\Omega^{2}(t)$. This means

$$
\phi(t)=-\frac{1}{24 \pi G \sqrt{C} V} \int d t \Omega^{2}(t)
$$

(13) now becomes

$$
L=-\frac{M}{2}\left[\dot{q}^{2}-\Omega^{2}(t) q^{2}\right]-\left(\frac{1}{2}\right) g(t)
$$

Now, the term $\frac{1}{2} g(t)=\sqrt{C} V \frac{d \phi}{d t}$ is a total time derivative and thus has no contribution to the equations of motion and hence ignorable. Then(15) becomes

$$
L=-\frac{M}{2}\left[\dot{q}^{2}-\Omega^{2}(t) q^{2}\right]
$$

So the problem of evolution of the scale factor in a homogeneous universe at early times (i.e. early compared to our present epoch but late compared to the big bang) may be studied by considering an oscillator with timedependent frequency.

Let us recollect what we have done. We started with a lagrangian where there was a dynamical degree of free$\operatorname{dom} q$ and a scalar field without a kinetic term. We then examine the lagrangian in a region where $q$ is small so that terms upto $O\left(q^{2}\right)$ are only kept and we have an oscillator.Interestingly, the role of the scalar field is transcribed into a time dependent frequency for our oscillator!

Thus, occurrence of parametric resonance becomes a distinct possibility.Parametric resonance in the early universe has been reported before in a different context. A theory of preheating and reheating after inflation was developed based on parametric resonance in the context of a scalar field $\chi$ coupled to an inflaton field [13].

It should be noted that the effect of the scalar field $\phi$ has been re-written as a time dependent frequency $\Omega(t)$, $\left(\Omega^{2}(t)=-24 \pi G \sqrt{C} V \frac{d \phi}{d t}\right)$. With this the lagrangian now looks like that of a time dependent oscillator and one of the solutions to this system is that of the parametric oscillator. First let us assume that the time dependent frequency is obtained as a relatively small sinusoidal perturbation from that of the ideal oscillator i.e.

$$
\Omega^{2}(t)=-24 \pi G \sqrt{C} V \frac{d \phi}{d t} \equiv \omega_{0}^{2}[1+f(t)]
$$

where $f(t)=h \cos \gamma t$, with the constant $h<<1$ and positive and $\omega_{0}^{2}>0$,i.e. $\omega_{0}$ real. Note that we will always be in the realm of small postive $t(t<1)$ and therefore

$$
\Omega^{2}(t)=-24 \pi G \sqrt{C} V \frac{d \phi}{d t}=\omega_{0}^{2}+\omega_{0}^{2} h \cos \gamma t
$$

and $\Omega^{2}(t)$ will always be positive. (This means that for consistency $\dot{\phi}$ has to be negative and this consistency is present in equation (17) below.) 
The physical motivation for taking $f(t)$ to be sinusoidal is as follows. A sinusoidal $f(t)$ can give growing solutions for $q$ and since $a=e^{q}$ we also get growing solutions for the scale factor which we know holds for an expanding universe. So the scalar field also helps in an inflation-like scenario. Note that the same scalar field can account for (a)growing solutions for a (shown below, equation (20)) (b)dark matter and dark energy as shown in [1] and (c)a simple scheme for estimating temperature fluctuations in certain epochs (Section 4). So the goal of building realistic models with a minimum number of scalar fields is also possible with this choice for $f(t)$.

Putting this $f(t)$ in equation (14) gives for small values of time $t$

$$
\phi(t) \approx \phi_{i n i}-\frac{\omega_{0}^{2}(1+h)}{24 \pi G \sqrt{C} V} t
$$

where $\phi_{i n i}$ is a constant of integration and may be identified with the initial value of $\phi$.

The excitation (forcing) term has amplitude $h$ and pe$\operatorname{riod} T_{\text {exc }}=\frac{2 \pi}{2 \omega}=\frac{\pi}{\omega}$. The unexcited (natural)period is $T_{n a t}=\frac{2 \pi}{\omega_{0}}$. Now solutions of linear ordinary differential equations with periodic coefficients [14] have following properties: (a) if the coefficients are periodic with period $\mathrm{T}$, then if $q(t)$ is a solution,so is $q(t+T)$

(b) Any solution $q(t)=A q_{1}(t)+B q_{2}(t)$ where $q_{1}(t), q_{2}(t)$ are two linearly independent solutions. $A, B$ come from initial conditions. Periodicity means that $q_{1}(t+T), q_{2}(t+$ $T)$ are also solutions and it follows that $q(t+T)=\lambda q(t)$. So $q(t)$ is periodic within a scaling parameter $\lambda$. Consider only growing or decaying solutions, not oscillations. Then $q(t+T)=e^{\mu T} q(t)$ where $\mu=\frac{\ln |\lambda|}{T}$. Finally, define $P(t)$ to be periodic such that $P(t+T)=P(t)$. Then

$$
q(t)=e^{\mu t} P(t)
$$

is the general form of the solution with the stability of the solution depending on the sign of $\mu$. Seek a solution of the general form $q(t)=A(t) \cos \left(\omega_{0}+\frac{1}{2} \epsilon\right) t+B(t) \sin \left(\omega_{0}+\frac{1}{2} \epsilon\right) t$. For $f(t)$ as given above and $\gamma=2 \omega_{0}+\epsilon, \epsilon<<\omega_{0}$, a solution of the general form [14] is

$$
\begin{aligned}
q(t) \equiv e^{\mu t} P(t)=A_{0} e^{\mu t} \cos \left(\omega_{0}+\frac{1}{2} \epsilon\right) t \\
+B_{0} e^{\mu t} \sin \left(\omega_{0}+\frac{1}{2} \epsilon\right) t
\end{aligned}
$$

where $\mu^{2}=\frac{1}{4}\left[\left(\frac{1}{2} h \omega_{0}\right)^{2}-\epsilon^{2}\right]$ and $A_{0}, B_{0}$ have to be determined from initial conditions. Here we have retained terms that are linear in $\epsilon$ and first order in $h$. Parametric resonance occurs in the range $-\frac{1}{2} h \omega_{0}<\epsilon<\frac{1}{2} h \omega_{0}$ on either side of $2 \omega_{0}$. The region of instability as well as the amplification coefficient $\mu$ are of the order of $h$. Parametric resonance also occurs when the frequency $\gamma$ is close to any value $\frac{2 \omega_{0}}{n}$ with $n$ integral. The region of instability and the amplification decreases rapidly with $n$.

For very small time scales the classical solutions for the scale factor of (16) can be written as (upto $O\left(t^{2}\right)$ terms):

$$
a_{c}(t)=e^{q_{c}(t)} \approx a_{i} e^{A t-B t^{2}}
$$

where $a_{i}=e^{A_{0}}$ is the initial value for $t=t_{i} \sim 0$, $A=\mu A_{0}+\omega B_{0}, B=\frac{1}{2} A_{0}\left(\omega^{2}-\mu^{2}\right)-B_{0} \mu \omega$ and $\omega \equiv \omega_{0}+\frac{1}{2} \epsilon$. So we have growing solutions for $a$ and not oscillating solutions. So there is no question of expanding and contracting scale factors. This solution is consistent with inflation. Taking the initial time as $t_{i}<<1$ and any observable epoch $t_{0} \sim 1$, the condition for an inflation like scenario, viz., $\frac{\dot{a}_{i}}{\dot{a}_{0}}<1$ is readily seen to be satisfied. The expansion here is similar to "chaotic inflation" 15] where the initial conditions of inflaton are distributed chaotically. For an inflaton potential $V(\phi)=\frac{1}{2} m^{2} \phi^{2}$, the inflaton field $\phi$ and the scale factor are given by [16]

$$
\begin{gathered}
\phi \approx \phi_{i}-\frac{m m_{\mathrm{P} l}}{2 \sqrt{3 \pi}} t \\
a \approx a_{i} \exp \left[2 \sqrt{\frac{\pi}{3}} \frac{m}{m_{\mathrm{P} l}}\left(\phi_{i} t-\frac{m m_{\mathrm{P} l}}{4 \sqrt{3 \pi}} t^{2}\right)\right]
\end{gathered}
$$

Comparing with (21), equations (17) and (20) will mimic the scenario of "chaotic inflation" with the following identifications:

$$
\begin{gathered}
\phi_{i}=\phi_{\text {ini }} ; \frac{\omega_{0}^{2}(1+h)}{\sqrt{C} V}=2 \sqrt{3 \pi}\left(\frac{m}{m_{\mathrm{Pl}}}\right) \\
A=\mu A_{0}+\left(\omega_{0}+\frac{1}{2} \epsilon\right) B_{0}=2 \sqrt{\frac{\pi}{3}}\left(\frac{m}{m_{\mathrm{Pl}}}\right) \phi_{i} \\
B=\frac{A_{0}\left[\left(\omega_{0}+\frac{1}{2} \epsilon\right)^{2}-\mu^{2}\right]}{2}-B_{0} \mu\left(\omega_{0}+\frac{1}{2} \epsilon\right)=\frac{1}{6} m^{2}
\end{gathered}
$$

The solution can also be made consistent with a radiation dominated era as follows. Using (20), the Hubble parameter is $H=\frac{\dot{a}_{c}}{a_{c}}=A-2 B t$, so that the energy density is

$$
\rho=\frac{3 H^{2}}{8 \pi G} \approx\left(\frac{3 m_{\mathrm{P} l}^{\frac{1}{2}}}{8 \pi}\right)\left(A^{2}-4 A B t+4 B^{2} t^{2}\right)
$$

For a radiation dominated era $\rho \sim a_{c}^{-4}$ with

$$
a_{c}^{-4} \approx a_{i}^{-4}-4 a_{i}^{-4} A t+a_{i}^{-4}\left(8 A^{2}+4 B\right) t^{2}
$$

Equating the coefficients of the $O(t)$ terms in (23) and (24) gives $A^{2}=B$ and this combined with the second and third equations of (22) imply the following for a radiation dominated era viz. $\phi_{i} \sim \frac{m_{\mathrm{P} l}}{8 \pi}$. This is consistent with "chaotic inflation" 16] where from considerations of the number of e-foldings required the bound on the initial 
value of the inflaton is obtained as $\phi_{i n i} \geq 3 m_{\mathrm{Pl}}$. Equating the coefficients of $O\left(t^{2}\right)$ terms in (23) and (24) and using $A^{2}=B$ gives $B=\frac{8 \pi}{m_{\mathrm{Pl}}^{2}} e^{-4 A_{0}}$. This combined with the last equation of (22) gives $A_{0} . B_{0}$ is then obtained from the second equation of (22) and $A=\sqrt{B}=\frac{m}{\sqrt{6}}$. (A consistency condition between $\mu$ and the last equation of (22) also exists as $\mu^{2}=(1 / 4)\left[(1 / 2)\left(h \omega_{0}^{2}-\epsilon^{2}\right]\right)$. Thus assuming that $t_{i}<<1 \sim 0$, one has

$$
\begin{gathered}
A_{0}=q\left(t=t_{i}\right)=\ln \left(\frac{2(3 \pi)^{\frac{1}{4}}}{\left(m m_{\mathrm{P} l}\right)^{\frac{1}{2}}}=\ln a\left(t_{i}\right)\right. \\
B_{0}=\frac{q\left(t=\frac{\pi}{2 \omega}\right)}{e^{\mu \pi / 2 \omega}}=\frac{\ln a\left(\frac{\pi}{2 \omega}\right)}{e^{\mu \pi / 2 \omega}}=\frac{m}{\sqrt{6} \omega}-\frac{\mu \ln a\left(t_{i}\right)}{\omega}
\end{gathered}
$$

Now $a\left(t=t_{i}\right) \neq 0$ so that $q_{i}=\ln a_{i}$ is still well defined. At some later time $t=t_{a}$, let $q=q_{a}$. Then the integration constants $A_{0}, B_{0}$ can be determined in terms of $q_{i}$ and $q_{a}$. Here $q_{i} \sim q\left(t_{i}\right)$ and $q_{a} \sim q\left(\frac{\pi}{2 \omega}\right)$. The inflaton mass must be $m \approx 10^{-6} m_{\mathrm{Pl} l}$ so as to fit the observed amplitudes of density perturbations by COBE satellite [17. Therefore, the domain we are considering has the initial value of the scale factor as $a\left(t_{i}\right) \sim \frac{2 \times 10^{3}(3 \pi)^{\frac{1}{4}}}{m_{\mathrm{P} l}}$.

\section{Fluctuations}

Let us now discuss how to estimate (quantum) fluctuations in this model. Given the structure of the lagrangian with canonical kinetic terms, this can always be done in principle. Write $q(t)=q_{c l}(t)+x(t)$ where $x(t)$ is the fluctuation from the classical value $q_{\mathrm{c} l}$ and $0 \leq x(t) \leq \infty$. So this corresponds to a time dependent oscillator on the half-plane [18], 19], 20], 21]. Then the quantum mechanical amplitude for the (log of) scale factor to evolve from the value $q_{a}$ at time $t_{a}$ to $q_{b}$ at time $t_{b}$ is given by [22]

$$
\left\langle q_{b}, t_{b} \mid q_{a}, t_{a}\right\rangle=\left\langle\ln a\left(t_{b}\right), t_{b} \mid \ln a\left(t_{a}\right), t_{a}\right\rangle=F\left(t_{b}, t_{a}\right) e^{\frac{i}{\hbar} S_{c l}}
$$

$S_{\mathrm{cl}}=\int_{t_{a}}^{t_{b}} L_{\mathrm{cl}} d t=\int_{t_{a}}^{t_{b}} d t\left[\frac{M}{2} \dot{q}_{\mathrm{cl}}^{2}-\frac{1}{2} \Omega^{2}(t) q_{\mathrm{cl}}^{2}\right]$. Here $\Omega(t)$ has been re-labelled as $\Omega(t) \rightarrow \sqrt{M} \Omega(t)$. (Factors of $\hbar$ and c will be put equal to unity at the end). $F\left(t_{b}, t_{a}\right)$ is calculated as follows [18]. The fluctuations $x(t)$ satisfy

$$
\ddot{x}+\Omega^{2}(t) x=0
$$

As $\Omega$ is real, we will have quasi-periodic solutions.

Here it may be mentioned that if one takes (say) the negative square root of $C$, (or equivalently $\omega_{0}^{2}<0$ ) then $\Omega^{2}<0$ and we will have quasi-exponential solutions for $x$ i.e. $x \approx A \exp \left[-\int|\Omega(t)| d t\right]+B \exp \left[+\int|\Omega(t)| d t\right]$. These solutions can be expected in the inflationary stage at which all second-order equations become elliptic. But in the present work all frquencies are real and we have only quasi-periodic solutions. (27):

Consider two independent quasi-periodic solutions of

$$
x_{1}(t)=\psi(t) \sin \zeta\left(t, t_{a}\right) ; x_{2}(t)=\psi(t) \sin \zeta\left(t_{b}, t\right)
$$

satisfying the boundary conditions

$$
x_{1}\left(t_{a}\right) \equiv x_{1 a}=0 \quad ; x_{2}\left(t_{b}\right) \equiv x_{2 b}=0
$$

where $\psi(t)$ satisfies the Ermakov-Pinney equation [23]

$$
\ddot{\psi}+\Omega^{2}(t) \psi-\psi^{-3}=0
$$

with $\zeta(t, s)$ defined as

$$
\zeta(t, s)=\nu(t)-\nu(s)=\int_{s}^{t} d t \psi^{-2}(t)
$$

$\psi(t)$ and $\nu(t)$ can be interpreted as the amplitude and phase of the time dependent oscillator. Then the fluctation factor is given by

$$
F\left(t_{b}, t_{a}\right)=\left(\frac{M \sqrt{\left(\dot{\nu}_{b} \dot{\nu}_{a}\right)}}{2 \pi i \hbar \sin \zeta\left(t_{b}, t_{a}\right)}\right)^{\frac{1}{2}}
$$

Classical solutions satisfying relevant boundary conditions are found, i.e. $q\left(t=t_{a}\right)=q_{a}, q\left(t=t_{b}\right)=q_{b}$ and the amplitude is

$$
\begin{gathered}
\left\langle q_{b}, t_{b} \mid q_{a}, t_{a}\right\rangle=\left\langle\ln a\left(t_{b}\right), t_{b} \mid \ln a\left(t_{a}\right), t_{a}\right\rangle \\
=\left(\frac{M \sqrt{\left(\dot{\nu}_{b} \dot{\nu}_{a}\right)}}{2 \pi i \hbar \sin \zeta\left(t_{b}, t_{a}\right)}\right)^{\frac{1}{2}}\left(\exp \left(\frac{i S_{\mathrm{cl}}^{+}}{\hbar}\right)-\exp \left(\frac{i S_{\mathrm{cl}}^{-}}{\hbar}\right)\right)
\end{gathered}
$$

where

$$
\begin{gathered}
S_{\mathrm{cl}}^{ \pm}=\left(\frac{\dot{\psi}_{b} q_{b}^{2}}{\psi_{b}}-\frac{\dot{\psi}_{a} q_{a}^{2}}{\psi_{a}}\right) \\
+\frac{1}{\sin \zeta\left(t_{b}, t_{a}\right)}\left(\left(\dot{\nu}_{b} q_{b}^{2}+\dot{\nu}_{a} q_{a}^{2}\right) \cos \zeta\left(t_{b}, t_{a}\right) \mp 2 \sqrt{\left(\dot{\nu}_{b} \dot{\nu}_{a}\right)} q_{b} q_{a}\right)
\end{gathered}
$$

Now assume $\dot{\nu}<<1$ i.e. the time rate of change of phase is small. Also note that the temperature of the background radiation in a homogeneous universe is inversely proportional to the scale factor i.e. $T\left(t_{a}\right) \equiv T_{a}=\frac{1}{a\left(t_{a}\right)}$ (in appropriate dimensionless units). Then to lowest orders of $\dot{\nu}$, one has the probability for the logarithm of scale factor ,or equivalently, logarithm of inverse temperature evolution as

$$
\begin{gathered}
P(b, a)=\left|\left\langle q_{b}, t_{b} \mid q_{a}, t_{a}\right\rangle\right|^{2}=\left(\frac{3 m_{\mathrm{P} l}^{2}}{\pi^{2} \hbar^{4} c}\right)\left(\frac{q_{a}^{2} q_{b}^{2}\left(\dot{\nu}_{b} \dot{\nu}_{a}\right)^{\frac{3}{2}}}{\sin \zeta^{3}\left(t_{b}, t_{a}\right)}\right) \\
\equiv\left|\left\langle\ln \left(\frac{1}{T_{b}}\right), t_{b} \mid \ln \left(\frac{1}{T_{a}}\right), t_{a}\right\rangle\right|^{2} \\
=\left(\frac{3 m_{\mathrm{P} l}^{2}}{\pi^{2}}\right)\left(\ln T_{a}\right)^{2}\left(\ln T_{b}\right)^{2} \frac{\left(\dot{\nu}_{b} \dot{\nu}_{a}\right)^{\frac{3}{2}}}{\sin \zeta^{3}\left(t_{b}, t_{a}\right)}
\end{gathered}
$$

where $\hbar^{4} c$ has been put equal to unity. 
Let us choose $\psi(t)=e^{\gamma t}$ where $0<\gamma<1$. Putting this in equation (30), using $\Omega^{2}(t)=-24 \pi G \sqrt{C} V \frac{d \phi}{d t}$ and solving for $\phi$ gives

$$
\begin{aligned}
& \phi(t)=\frac{1}{24 \pi G \sqrt{C} V}\left[C_{0}+\gamma^{2} t+\frac{1}{4 \gamma} e^{-4 \gamma t}\right] \\
& \approx\left(C_{0}+\frac{1}{96 \gamma \pi G \sqrt{C} V}\right)-\frac{\left(1-\gamma^{2}\right)}{24 \pi G \sqrt{C} V} t
\end{aligned}
$$

$C_{0}$ is a constant of integration. Therefore the choice of $\psi(t)=e^{\gamma t}$ is consistent with what has been discussed before (viz. equations (17), (22)) with the following identifications:

$$
\phi_{\mathrm{i} n i} \equiv C_{0}+\frac{1}{96 \gamma \pi G \sqrt{C} V} \quad ; \quad \omega_{0}^{2}(1+h)=1-\gamma^{2}
$$

Note that bounds on $\sqrt{C} V$ can be obtained in terms of $C_{0}, G, \gamma$ and $\phi_{i n i} \geq 3 m_{\mathrm{Pl}}$ [16 (discussion after equation (24)). Further, with this choice for $\psi(t)$, one has the following relations:

$\nu(t)=-\frac{1}{2 \gamma} e^{-2 \gamma t} ; \sin \zeta(t, s)=\sin (\nu(t)-\nu(s)) \approx t-s$

For small times, then the probability for the logarithm of inverse temperature evolution, (35) becomes

$$
\begin{gathered}
P(b, a)=\left|\left\langle\ln \left(\frac{1}{T_{b}}\right), t_{b} \mid \ln \left(\frac{1}{T_{a}}\right), t_{a}\right\rangle\right|^{2} \\
\approx\left(\frac{3 m_{\mathrm{P} l}^{2}}{\pi^{2}}\right) \frac{\left(\ln T_{a}\right)^{2}\left(\ln T_{b}\right)^{2}\left(1-3 \gamma\left(t_{a}+t_{b}\right)\right)}{\left(t_{b}-t_{a}\right)^{3}}
\end{gathered}
$$

Let us estimate the physical domain of validity of this expression. By physical domain I mean the times $t_{a}, t_{b}$ and the temperatures $T_{a}, T_{b}$. As already stated in the introduction (section 1), all our times have to be understood as $t \equiv \frac{t}{t_{0}}$. The requirement that the $P(b, a) \geq 0$ and $0<\gamma<1$ means

$$
t_{a}+t_{b} \geq \frac{1}{3}
$$

Again the requirement $P(b, a) \leq 1$ implies

$$
t_{b}-t_{a} \leq\left(\frac{3 m_{\mathrm{P} l}}{\pi}\left[\ln T_{a}\right]\left[\ln T_{b}\right]\right)^{\frac{2}{3}}
$$

Combining (40) and (41) gives

$$
\begin{aligned}
t_{a} & \geq \frac{1}{6}-\frac{1}{2}\left(\frac{3 m_{\mathrm{Pl} l}}{\pi}\left[\ln T_{a}\right]\left[\ln T_{b}\right]\right)^{\frac{2}{3}} ; \\
t_{b} & \geq \frac{1}{6}+\frac{1}{2}\left(\frac{3 m_{\mathrm{P} l}}{\pi}\left[\ln T_{a}\right]\left[\ln T_{b}\right]\right)^{\frac{2}{3}}
\end{aligned}
$$

Now combining (42) with the requirement that all estimates are valid only for $t_{a}>0$ means that numerically the following must hold (in appropriate dimensionless units) i.e.

$$
\left[\ln T_{a}\right]\left[\ln T_{b}\right] \leq \frac{\pi}{9 \sqrt{3} m_{\mathrm{Pl}}}
$$

Therefore, the formalism described here has given a new way of describing fluctuations of the background temperature. This estimate can be made as long as one is in the temporal region where the lagrangian (16) is valid and the conditions embodied in equations (40) - (43) hold true. Note that there exist functional time dependent parameters in the quantum amplitude, viz., $\dot{\nu}\left(t_{a}\right), \dot{\nu}\left(t_{b}\right), \zeta\left(t_{b}, t_{a}\right)$ which are related to solutions of the Ermakov-Pinney equation. Now, the bounds on these functions may be estimated from phenomenological observations of satellite data if the fluctuations of the background temperature can ever be accurately determined.

\section{Conclusion}

A unified formalism has been set up with a $k$-essence lagrangian containing canonical kinetic terms which provides a framework for estimating quantum fluctuations of the temperature in certain epochs. In an earlier epoch in the universe defined by the scale factor being very small compared to the present epoch but very large compared to the big bang and inflationary epoch (so that one is already in an expanding and flat universe), this lagrangian is similar to an oscillator with time dependent frequency. A sinusoidal perturbation from an ideal oscillator frequency leads to growing classical solutions for the scale factor and can be made consistent with the scenario of chaotic inflation as well as with a radiation dominated universe at a certain epoch by suitable choice of parameters. As the time dependent frequency is basically related to a scalar field, it can also be taken to be similar to the "inflaton". Although (as stated in the beginning) we are strictly away from the inflationary scenario, we would like to have the scalar field to be as far as possible similar to that needed for inflation. This does away with the need for multiple scalar fields to account for specific features of evolution of the universe. What has been shown here is that if the "inflaton" is given an opportunity to influence the evolution for longer times, it can also help in contributing to the dark matter and dark energy scenario.

Quantum fluctuations of the log of inverse temperature can be estimated in principle for a certain physical domain characterised by the epochs and the respective temperatures satisfying certain bounds. In this region the expressions for the quantum fluctuations are valid. At much later times, dark matter and dark energy can be be accounted for as shown in [1].Establishing a formalism for quantum fluctuations of the (inverse) temperature has been possible in this model owing to the presence of standard (canonical) kinetic terms in the lagrangian so that 
the usual prescriptions are applicable without ambiguity. This is the first model of this type.

\section{Acknowledgments}

The author thanks the referee for extremely illuminating suggestions for improving the manuscript.

The author also thanks the Centre For Astroparticle Physics and Space Science, Bose Institute, Kolkata, for a sabbatical tenure during which this work was done and Sandip Chakrabarty for illuminating discussions.

*debashis@bose.res.in

[1] D.Gangopadhyay and S. Mukherjee,Logarithm of the scale factor as a generalised coordinate in a lagrangian for dark matter and dark energy, Phys. Lett.B665 121 (2008) arXiv:0710.5366.

[2] R.J.Scherrer, Purely kinetic k-essence as unified dark matter Phys.Rev.Lett. 93011301 (2004) [astro-ph/040231].

[3] C.Armendariz-Picon, T.Damour and V.Mukhanov, $k$ Inflation, Phys.Lett. B458 209 (1999) hep-th/9904075; J.Garriga and V.F.Mukhanov,Perturbations in $k$ inflation, Phys.Lett. B458 219(1999) hep-th/9904176.

[4] T.Chiba, T.Okabe and M.Yamaguchi, Kinetically driven quintessence, Phys.Rev. D62 023511 (2000) astro-ph/9912463;

C.Armendariz-Picon, V.Mukhanov and P.J.Steinhardt, $A$ dynamical solution to the problem of a small cosmological constant and late time cosmic acceleration, Phys.Rev.Lett. 854438 (2000) astro-ph/0004134;

C.Armendariz-Picon, V.Mukhanov and P.J.Steinhardt, Essentials of k-essence, Phys.Rev. D63 103510 (2001) astro-ph/0006373;

T.Chiba, Tracking k-essence,Phys.Rev. D66 063514 (2002) astro-ph/0206298.

[5] L.P.Chimento, Extended tachyon field, Chaplygin gas and solvable k-essence cosmologies,Phys.Rev. D69 123517 (2004) astro-ph/0311613.

[6] M.Gasperini and G.Veneziano, The pre-big bang scenarion in string cosmology, Phys.Rep. 3731 (2003) hep-th/0207130;

K.I.Maeda,Towards the Einstein-Hilbert action via conformal transformation, Phys.Rev. D39 3159 (1989).

[7] V.Sahni,Dark matter and dark energy, Lect.Notes Phys. 653141 (2004) astro-ph/0403324; 843111 (2006) astro-ph/0602117;

T.Padmanabhan,Dark energy: mystery of the millenium, AIP Conf.Proc. 861179 (2006) astro-ph/0603114;

T.Padmanabhan,Dark energy and gravity, Gen.Rel.Grav. 40529 (2007) arXiv:0705.2533;

E.J.Copeland,M.Sami and S.Tsujikawa, Dynamics of dark energy, Int.Jour.Mod.Phys. D15 1753 (2006) hep-th/0603057.

[8] P.J.E.Peebles and B.Ratra, The cosmological constant and dark energy, Rev.Mod.Phys. 75559 (2003) ;

T.Padmanabhan, Cosmological constant-the weight of the vacuum, Physics Reports $\mathbf{3 8 0} 235$ (2003) hep-th/0212290.

[9] M.Malquarti,E.J.Copeland,A.R.Liddle and M.Trodden, $A$ new view of k-essence, Phys.Rev. D67 123503 (2003) astro-ph/0302279.
M.Malquarti,E.J.Copeland and A.R.Liddle, K-essence and the coincidence problem, Phys.Rev. D68 023512 (2003) astro-ph/0304277;

L.Mingzhe and X.Zhang, K-essence leptogenesis, Phys.Lett. B573 20 (2003) hep-ph/0209093;

J.M.Aguirregabiria,L.P.Chimento and R.Lazkoz, Phantom k-essence cosmologies, Phys.Rev. D70 023509 (2004) astro-ph/0403157.

[10] L.P.Chimento and R.Lazkoz,Atypical k-essence cosmologies, Phys.Rev. D71 023505 (2005) astro-ph/0404494; L.P.Chimento,M.Forte and R.Lazkoz,Dark matter to dark energy transition in $k$-essence cosmologies, Mod.Phys.Lett. A20 2075 (2005) astro-ph/0407288;

R.Lazkoz, Rigidity of cosmic acceleration in a class of $k$ essence cosmologies, Int.Jour.Mod.Phys. D14 635 (2005) gr-qc/0410019;

H.Kim, Brans-Dicke scalar field as a unique $k$-essence, Phys.Lett. B606 223 (2005) astro-ph/0408154;

J.M.Aguirregabiria,L.P.Chimento and R.Lazkoz,Quintessence as k-essence, Phys.Lett. B631 93 (2005) astro-ph/0411258;

H.Wei and R.G.Cai, K-chamelion and the coincidence problem, Phys.Rev. D71 043504 (2005) hep-th/0412045;

C.Armendariz-Picon and E.A.Lim,Haloes of k-essence, JCAP 05087 (2005) astro-ph/0505207.

[11] L.R.Abramo and N.Pinto-Neto, On the stability of phantom k-essence theories, Phys.Rev. D73 063522 (2006) astro-ph/0511562;

A.D.Rendall, Dynamics of k-essence, Class.Quant.Grav. 231557 (2006) gr-qc/0511158.

[12] A.Sen, Rolling Tachyon, JHEP 0204048 (2002) hep-th/0203211.

[13] L.Kofman,A.Linde and A.Starobinsky, Reheating after inflation, Phys.Rev.Lett. $73 \quad 3195 \quad$ (1994) hep-th/9405187;

L.Kofman,A.Linde and A.Starobinsky, Towards the theory of reheating after inflation, Phys.Rev. D56 3258 (1997) hep-ph/9704452.

[14] L.D.Landau and E.M.Leifshitz, Mechanics, Vol.1, Course of Theoretical Physics,(Pergamon Press, Oxford, 1976).

[15] A.Linde, Phys.Lett. 129B 177 (1983);

A.Linde, Particle Physics and Inflationery Cosmology (Harwood,Chur,1990).

[16] Shinji Tsujikawa, Introductory Review of Cosmic Inflation hep-ph/0304257.

[17] A.R.Liddle and D.H.Lyth in Cosmological Inflation and Large Scale Structure (Cambridge University Press, 2000).

[18] D.C.Khandekar and S.V.Lawande,Phys.Rep.137 115 (1986).

[19] H.Ezawa,J.R.Klauder and L.A.Shepp, J.Math.Phys. 16 783 (1975).

[20] B.Simon, J.Functional Analysis and Applications 14295 (1973).

[21] T.E.Clark, R.Menikoff and D.H.Sharp, Phys.Rev. D22 3012 (1980).

[22] R.P.Feynman and A.R.Hibbs, Quantum Mechanics and Path Integrals (McGraw Hill, New York, 1965).

[23] V.P.Ermakov, Univ.Izv.Kiev 201 (1880); E.Pinney, Proc.Am.Math.Soc.1 681 (1950). 


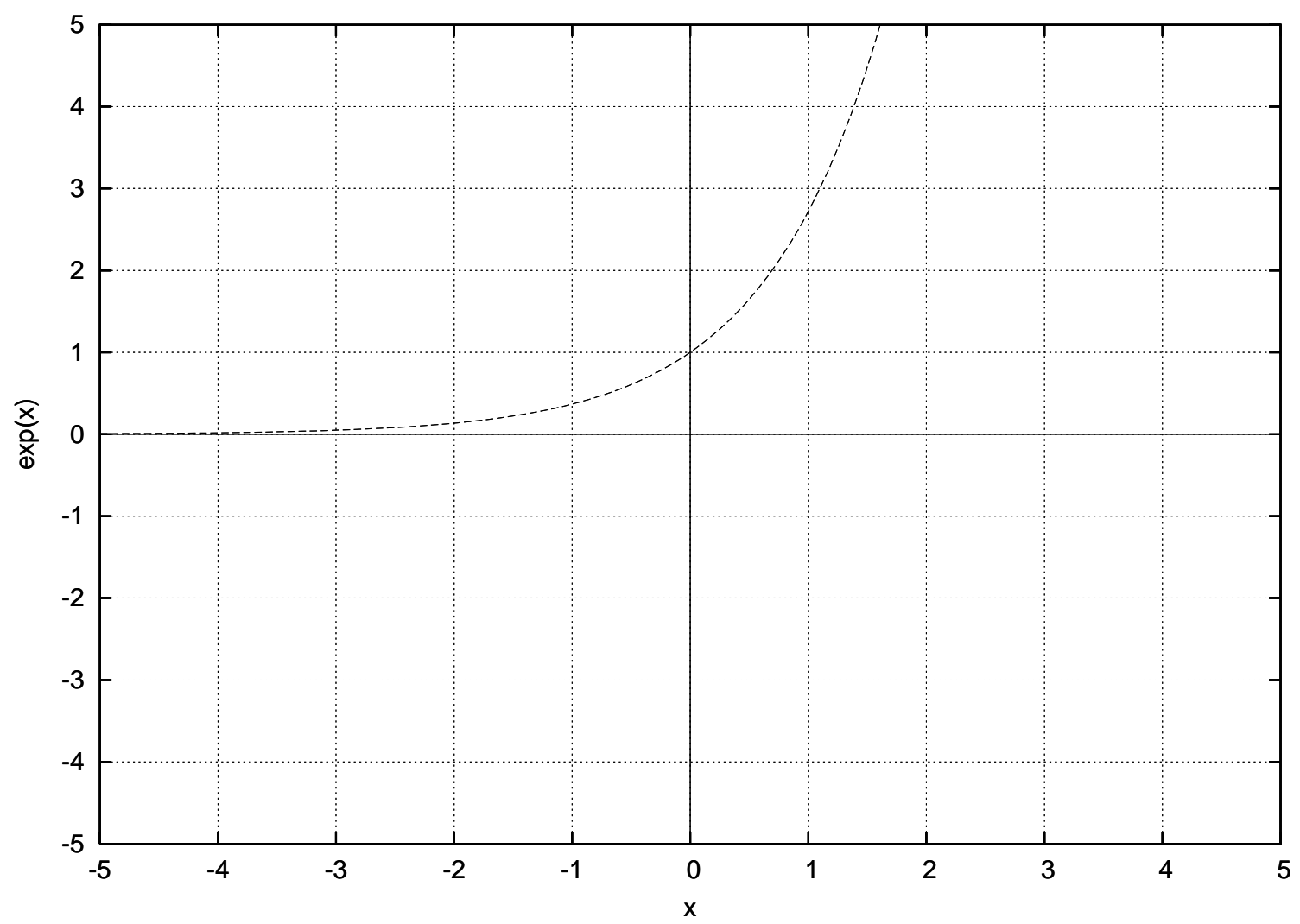

